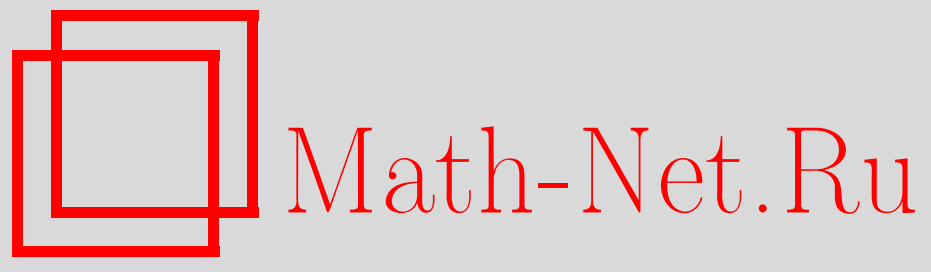

В. П. Маслов, Фазовые переходы нулевого рода и квантование закона Ципфа, ТМФ, 2007, том 150, номер 1, 118-142

DOI: https://doi.org/10.4213/tmf5969

Использование Общероссийского математического портала Math-Net.Ru подразумевает, что вы прочитали и согласны с пользовательским соглашением http://www . mathnet.ru/rus/agreement

Параметры загрузки:

IP : 54.164 .48 .24

26 апреля 2023 г., 14:32:33

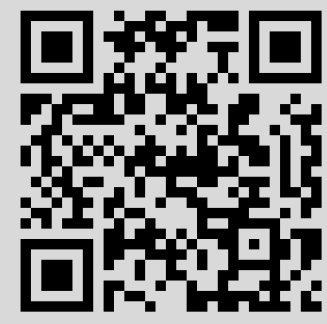




\title{
ФАЗОВЫЕ ПЕРЕХОДЫ НУЛЕВОГО РОДА И КВАНТОВАНИЕ ЗАКОНА ЦИПФА
}

\begin{abstract}
Исследуется происхождение выбросов в АЭС после аварии или после выработки ресурса с точки зрения термодинамического осреднения микропроцессов распада. Генезис выбросов в АЭС трактуется как новое термодинамическое явление - фазовый переход нулевого рода. Эта постановка вопроса приводит к задаче, относящейся к вероятностной теории чисел. Доказана соответствующая теорема, приводящая к квантованию закона Ципфа для повторяемости фазового перехода нулевого рода с разными значениями скачка термодинамического потенциала Гиббса. Вводится понятие дырочной размерности.
\end{abstract}

Ключевые слова: Чернобыльская АЭС, вероятностная теория чисел, распределение Бозе-Эйнштейна, квантование, закон Ципфа, термодинамика, отрицательная размерность.

$$
\begin{array}{r}
A x, \text { Толя, Толя, ты ли, ты ли ... } \\
\text { С. Есенин }
\end{array}
$$

Один из основоположников теории вероятностей А. Н. Колмогоров наметил путь пересмотра теории вероятностей с точки зрения дискретного подхода [1]-[4]. В таком подходе отношение числа выпадений к числу испытаний не обязательно имеет предел. Кроме того, понятие случайности определено Колмогоровым как "максимально сложное", что не соответствует его обывательскому смыслу. Но на самом деле, если каждое случайное событие объяснять детерминистическим образом, алгоритм возникновения этого события будет очень сложным, а расшифровка (декодирование) этого алгоритма потребует очень длинного кода. Становятся популярными и другие подходы к теории вероятностей [5].

\section{1. ТЕРМОДИНАМИЧЕСКИЕ ПРОЦЕССЫ В АЭС ПОСЛЕ АВАРИИ ИЛИ ПОСЛЕ ВЫРАБОТКИ РЕСУРСА}

После того как атомные станции исчерпали свой ресурс, их нельзя остановить. Они продолжают жить. И этот факт “долгожительства" означает, что внутри АЭС, отслужившей свой век или после аварии, происходят известные микропроцессы распада, которые мы должны изучить с точки зрения макрофизики, т.е. применить к ним осредненный термодинамический подход.

* Московский государственный университет, Москва, Россия. E-mail: v.p.maslov@mail.ru 
До сих пор мы были знакомы с несколькими авариями, самой крупной из которых была авария на 4-м блоке Чернобыльской АЭС. Сейчас выработала свой срок Ленинградская АЭС (и дала выброс). Что с ней делать дальше? Решили продлить ее эксплуатацию. На грани окончания срока действия находятся французские АЭС. Как они будут вести себя после остановки? Ведь экспериментов в лаборатории не поставишь. Это совершенно новое явление, и мы поставлены перед проблемой, которая никогда раньше не возникала: понять, что заключено в "черном ящике" очень медленно умирающей атомной станции после аварии или после истечения ее срока действия. Не ставит ли этот "черный ящик", опасный для существования всего живого, совершенно новые задачи перед статистической физикой и наукой о ее средних (термодинамикой)?

Весной 1986 г. меня пригласили собрать и возглавить группу математиков для участия в ликвидации последствий аварии 4-го блока Чернобыльской АЭС. Огромная ответственность, сжатые сроки, неожиданность некоторых обнаруженных явлений потребовали громадного напряжения наших творческих сил и перевернули мое представление о математике как о науке в целом. В 1987 г. мы опубликовали наш отчет в книге [6], но на главные вопросы ответы найдены не были. Стало ясно, что современная математика должна быть пересмотрена для того, чтобы можно было обобщить и углубить термодинамику, теорию вероятностей и математическую физику - дисциплины, необходимые для решения проблем, возникающих в АЭС после аварий.

Поскольку оказаться внутри разлома и проводить там измерения невозможно, то результаты измерений оказываются осредненными, термодинамическими. Хотя в некоторых отсеках излучение может практически отсутствовать, опасные пятна внутри разлома будут попадаться достаточно случайно. Для объяснения ряда факторов приходится произвести некоторую ревизию и обобщение термодинамической науки применительно к непредвиденным физическим явлениям. Так как в станции после аварии или после того, как она исчерпает свой ресурс, процессы ядерного деления продолжаются, мы должны оценить эту ситуацию с точки зрения термодинамической науки, считая время "жизни” станции бесконечно большим.

Вообще, пересмотр основных понятий классической термодинамики уже несколько запоздал, так как после открытия сверхтекучести представление о том, что при нулевой температуре все молекулы останавливаются, было опровергнуто опытом. Поэтому необходимо было изучить явление сверхтекучести с физической точки зрения и с точки зрения математико-физического обобщения. Замечательные результаты школ Ландау и Боголюбова необходимо обобщить так, чтобы их можно было применить к совсем, казалось бы, противоположной ситуации перегрева при аварии АЭС. Работы физиков, обладающих гениальной интуицией, нуждаются в последующих математико-физических и даже, может быть, философских обобщениях теоретиков такого уровня, как Фок, фон Нейман, Вигнер и другие.

В письмах Колмогорова приводится мнение Капицы относительно сверхтекучести. Колмогоров пишет: "П. Л. [Капица] сообщил следующее: еще перед началом войны им [Капицей] были произведены опыты для проверки теории Ландау. Опы- 
ты эти дали результаты (еще не опубликованные), решительно опровергающие все построения Ландау... Очевидно, задержка опубликования опытных данных для того и устроена, чтобы Ландау свою теорию не успел поправить (так как известно, что "ему ничего не стоит изготовить теорию под любые данные!") ([7], с. 594). Последние кавычки, очевидно, относятся к словам Капицы.

На самом деле моментально откликнуться на новые опытные данные - это талант и шаг к истине. А уже связать воедино разные концепции сверхтекучести, классическую термодинамику, теорию осреднений (теорию вероятностей) и данные аварий - это уже задача специалиста в области математической физики. У меня был для этого огромный стимул, поскольку последние 20 лет, прошедшие после Чернобыльской аварии, мне не давали покоя те проблемы, которые не удалось решить тогда, а те переживания, которые я испытал, подписывая разрешение на закрытие саркофага крышкой без полной уверенности, постоянно мучили меня даже во сне. Каждый очередной выброс различных АЭС возвращал меня к этим проблемам.

Для решения этих проблем статистическая физика и наука о ее осреднении термодинамика, с одной стороны, и математическая статистика и наука о ее осреднении - теория вероятностей, с другой стороны, должны быть объединены в одну науку. Однако современный уровень теории вероятностей недостаточен для описания процессов, происходящих в АЭС. Если вероятность аварии пренебрежимо мала, но последствия аварии грандиозны и страшны, то пренебрегать даже "бесконечно" малой вероятностью нельзя. Второй момент следующий. Приведу математический пример игры в орла и решку. Какова бы ни была предыстория выпадения орла или решки до данного момента, вероятность выпадения орла или решки в данный момент равна $1 / 2$. Однако, как пишет известный английский физик Пайерлс про рулетку (вместо орла и решки - черное и красное), сомнительно, чтобы "нашелся хоть один игрок, который, рискнув на "черном" один раз, продолжал ставить весь свой выигрыш постоянно на тот же номер. . . Вероятность появления такой “черной” серии настолько мала, что ее следует полностью игнорировать” ([8], с. 126). Как это согласуется с тем, что вероятность от предыстории не зависит?

Эти соображения очень близки к понятию “общего положения" в топологии, где "общим положением", например, кривой на плоскости относительно проецирования на ось абсцисс называется такое расположение кривой, при котором "мощность" множества близких кривых с теми же свойствами много больше "мощности" множества кривых с другими свойствами. А это похоже на те соображения, которые привел Больцман в ответ на возражение Цермело: если из многообразия всех возможных начальных состояний взять произвольным образом одно, то крайне маловероятно, что оно окажется с убывающей энтропией. Это понятие, близкое к устойчивости, не относится к математической теории вероятностей, но оно обязательно должно присутствовать в новой ревизованной теории вероятностей, поскольку отвечает вышеуказанному классу проблем.

Отметим, что общеизвестных понятий математики, таких как необходимые и достаточные условия, недостаточно для решения конкретных проблем АЭС после аварии. Например, “необходимые условия" для построения саркофага, чтобы выброс 
обязательно случился, написать можно, а сформулировать “достаточные условия", чтобы на 100\% гарантировать, что выброса не будет, более чем сложно.

Как выбрать из множества равноценных конструкций наиболее надежную, “устойчивую”? Эта проблема близка к тому “закону", который приводит Пайерлс, и к понятию общего положения. Общий термодинамический подход, учитывающий соотношение времени жизни и времени релаксации к квазиравновесному состоянию, показывает, что между такими явлениями, как фонтанирование сверхтекучего гелия в опыте Аллена-Джонса и выбросами в АЭС, есть общее - это не замеченный физиками-теоретиками фазовый переход нулевого рода (см. ниже), когда испытывает скачок не только энтропия (или внутренняя энергия, т.е. фазовый переход первого рода), но и термодинамический потенциал Гиббса. Момент, в который это может произойти, можно определить из соответствующих уравнений состояния. Этот эффект существенно отличается от известной картины фазового перехода первого рода.

Гидродинамический, достаточно медленный процесс был выявлен и в грубом приближении исследован в нашем отчете.

Существовало мнение, что твэлы ${ }^{1)}$ будут расплавлять все, что находится под ними, и проходить сквозь землю в подземные воды, как утюг проходит сквозь лед. Это могло вызвать страшную экологическую катастрофу. Чтобы этого избежать, еще до наших расчетов была сооружена огромная бетонная подушка под завалом, которая должна была препятствовать проникновению твэлов под землю. Однако эта огромная работа была сделана по существу напрасно, так как расчеты показали, что нежелательного эффекта можно избежать. Мы с покойным В. П. Мясниковым установили, что к данной задаче применимо уравнение типа Дарси, т.е. фильтрационное уравнение. Для ее решения были поставлены эксперименты и проведен большой объем математических расчетов. Оказалось, что даже в случае фильтрации небольшой поддув снизу вследствие разницы давления внизу и наверху завала снижает температуру под твэлом. Это подобно "каминному эффекту", когда каминная решетка не нагревается несмотря на очень высокую температуру горящих углей. На практике это подтвердилось: ни один твэл не достиг бетонной подушки. Трехмерные расчеты, учитывающие конструкцию саркофага и сам гидродинамический процесс "конвекции" в фильтрационной среде, были уточнены позднее.

\section{2. ФАЗОВЫЕ ПЕРЕХОДЫ}

\section{1. Общие соображения о фазовом переходе нулевого рода.}

2.1.1. Основные макропараметры термодинамики. Термодинамика изучает установившиеся процессы, когда независимо от своего начального состояния система приходит в состояние, которое в дальнейшем уже не меняется. Если же все-таки такое состояние меняется, то изменение является термодинамическим только в том случае, если процесс совершается чрезвычайно медленно [9]. Иначе говоря, мы бесконечно мало меняем состояние системы и ждем, пока она не придет в равновесие и не будет зависеть от начального состояния.

\footnotetext{
1) Твэлы - тепловыделяющие элементы.
} 
Таким образом, здесь незримо присутствует макропараметр - время, т.е. мы рассматриваем систему через огромные промежутки времени. С другой стороны, термодинамика является предельной макроскопической теорией, получающейся из микроскопической статистической физики при числе частиц $N \rightarrow \infty$. Следовательно, имеются два макропараметра и от их соотношения многое зависит. В свою очередь, классическая статистическая физика является пределом квантовой статистики при $h \rightarrow 0$ ( $h$ - параметр Планка). Таким образом, “сталкиваются” уже три макропараметра: масштаб времени, число частиц и $1 / h$.

Термодинамика рассматривает часто и достаточно большие периоды времени, за которые лишь часть системы приходит в равновесие, т.е. предметом изучения является так называемое локальное равновесие. Например, при нагревании плазмы магнитным полем через некоторое большое наблюдаемое время для ионов устанавливается распределение Максвелла, и лишь через существенно большее время вся система приходит в термодинамическое равновесие.

2.1.2. Постулат Гиббса. Имеет место постулат Гиббса, который в квантовой статистике звучит следующим образом.

Пусть система характеризуется оператором Гамильтона $\widehat{H}_{N}$, где $N$ - число частиц в гильбертовом пространстве $\Phi$ (в частности, в пространстве Фока). Пусть оператор $\widehat{H}_{N}$ имеет неотрицательный дискретный спектр $\lambda_{0}(N), \lambda_{1}(N), \ldots, \lambda_{n}(N), \ldots$. Тогда свободная энергия, зависящая от температуры $\theta$, определяется как $E=$ $\theta \ln \left(\sum_{n=0}^{\infty} e^{-\lambda_{n} / \theta} \delta_{n}\right)$, где $\delta_{n}-$ кратность собственного значения $\lambda_{n}=\lambda_{n}(N)$.

Отметим, что в постулате Гиббса на самом деле присутствует скрытый параметр, а именно $E=\theta \ln \left[\lim _{M \rightarrow \infty} \sum_{n=0}^{M} e^{-\lambda_{n} / \theta} \delta_{n}\right]$. Это один из важнейших моментов, так как пределы $\lim _{M \rightarrow \infty}$ и $\lim _{N \rightarrow \infty}$ не коммутируют! Оказывается, сначала нужно вычислить предел при $M \rightarrow \infty$, а затем при $N \rightarrow \infty$.

Когда речь идет о локальном равновесии, то в формуле Гиббса, как правило, рассматривается некоторое подмножество $\left\{\lambda_{n^{\prime}}\right\}$ множества $\left\{\lambda_{n}\right\}$. Это нужно делать, исследуя вышеупомянутую задачу о распределении ионов в плазме при нагревании. Это же делается в задаче о спиновых стеклах и ряде других физических задач. У нас постулат Гиббса доказан - его уточнение следует из доказанной ниже теоремы (см. раздел 3), относящейся к теории множеств.

2.1.3. Оператор трения. При температуре $\theta=0$ формула Гиббса дает нижнее собственное значение, которое в физике называется основным состоянием.

Суть заключается в том не сформулированном четко законе, который Боголюбов и другие называют “энергетической выгодностью”. Энергетически выгодна более низкая энергия. Например, если мы возмущаем систему некоторым оператором $\widehat{V}:(\Phi \rightarrow \Phi)$, который достаточно мал, то матрица перехода из состояния $\lambda_{n}$ в состояние $\lambda_{m}$ определяется матричным элементом $\left(\Psi_{n} \widehat{V} \Psi_{m}^{*}\right)$, где $\Psi_{n}$ и $\Psi_{m}-$ собственные функции, отвечающие состояниям $\lambda_{n}$ и $\lambda_{m}$, соответственно (см. [10]). Квадрат этого матричного элемента есть вероятность перехода из состояния с энергией $\lambda_{n}$ в состояние с энергией $\lambda_{m}$. Но если $\lambda_{n}<\lambda_{m}$, то этот переход энергетически невыгоден и поэтому с точки зрения статистической физики и термодинамики неосуществим, т.е. с математической точки зрения вероятность его надо положить равной нулю. 
Тогда матрица перехода будет несамосопряженной с нулями выше диагонали. Это означает учет трения. Маятник колеблется и при наличии трения в конце концов остановится, т.е. попадет в основное состояние.

2.1.4. Обычные фазовые переходы. Производная свободной энергии $E=E(\theta)$ по $\theta$ называется энтропией, и когда в некоторой точке $\theta_{0}$ она испытывает скачок, то говорят, что осуществляется фазовый переход первого рода. В случае, когда ее вторая производная испытывает скачок, происходит фазовый переход второго рода, и т.д. Но разумеется, испытывает скачок не сама функция, а ее главный член асимптотики при $N \rightarrow \infty$. Этот “скачок" может быть иногда реально на эксперименте растянут по времени, но мы условились рассматривать изменения на достаточно больших промежутках времени. В этих промежутках могут происходить не термодинамические, а динамические процессы.

2.1.5. Метастабильное состояние. Рассмотрим простой пример квазиклассического приближения одномерного уравнения Шредингера

$$
-h^{2} y_{n}^{\prime \prime}+u(x) y_{n}=\lambda_{n} y_{n}, \quad y_{n}(x) \in L_{2}, \quad h \ll 1,
$$

где $u(x)=\left(x^{2}-1\right)^{2}+q x, q>0$ - константа. В классической механике это уравнение описывает две впадины, разделенные между собой барьером. Если частица находится на дне правой, менее глубокой впадины, то она не “перекатится" во вторую, более низкую, если только барьер не исчезнет.

$\mathrm{C}$ точки зрения постулата Гиббса при $\theta \rightarrow 0$ частица должна оказаться в более глубокой впадине. Вместе с тем очевидно, что если $h \ll 1$, то в менее глубокой впадине она будет находиться очень длительное время. В формуле Гиббса в этом случае нужно проводить суммирование по подмножеству собственных значений, отвечающих менее глубокой впадине, собственные функции которых стремятся к нулю при $h \rightarrow 0$ вне этой впадины. При этом температура $\theta$ должна быть не слишком велика, чтобы собственные значения выше барьера не играли бы роль в формуле Гиббса. Такое состояние в локальном минимуме потенциальной ямы $u(x)$ представляет собой пример метастабильного состояния.

Если мы рассматриваем элемент матрицы перехода с нижнего уровня, отвечающего менее глубокой впадине, на самый нижний уровень $\lambda_{0}$ (на дне глубокой впадины), то он оказывается экспоненциально мал по $h$, переход же на высокие уровни запрещен в силу закона "энергетической выгодности" (т.е. мы рассматриваем возмущение "трения").

2.1.6. Сверхтекучесть. Как показал Боголюбов [11], использовавший некоторые идеи Ландау (см. [12], сноска на с. 219), сверхтекучесть - это не движение жидкости, не динамика, а состояние, как, например, лед и пар для воды. Оно отвечает некоторому метастабильному состоянию системы, переход из которого в нормальное почти запрещен: он экспоненциально мал при $N \rightarrow \infty$. Боголюбов доказал это строго при условии периодичности для системы уравнений Шредингера, иначе говоря на торе. Спектр сверхтекучих скоростей (уровней энергии, отвечающих соответствующим импульсам) дискретный. Это сразу отличает состояние сверхтекучести от гидродинамики жидкости. В пределе, когда радиус тора устремляется к бесконечности, 
спектр отнюдь не переходит в непрерывный, как это обычно бывает, а становится всюду плотным, точечным.

С физической точки зрения, на мой взгляд, тот факт, что спектр является в пределе всюду плотным точечным, легко объясним. Действительно, если система находится в состоянии со сверхтекучей скоростью $v$, то переход ее к большей скорости запрещен законом "энергетической выгодности", а переход к сколь угодно меньшей скорости запрещен тем, что уменьшение скорости противоречит понятию сверхтекучести. С математической точки зрения чем радиус тора больше, тем менее вероятен переход из одного состояния в другое (вероятность экспоненциально меньше).

\subsection{7. Примеры фазовых переходов нулевого рода.}

ПримеР 1. Если бы мы могли создать такие “стерильные” условия, чтобы вода перегревалась выше максимальной температуры, которая определяется окончанием метастабильного состояния на графике температура-энтпропия, и тогда после превышения максимальной температуры произошел бы неизбежный фазовый переход не в результате внешних воздействий, а в силу того, что метастабильное состояние при такой температуре вообще отсутствует, то в этом случае согласно нашему определению произошел бы фазовый переход нулевого рода. Однако на эксперименте достичь такого состояния, по-видимому, невозможно. При этом на самом деле скачок испытывает не только потенциал Гиббса, в этой точке возникло бы еще и ветвление вида $\sqrt[6]{x}[13]$. Но как отмечалось выше, создать экспериментально такой барьер для метастабильного состояния, чтобы оно продержалось до своего максимума, невозможно: можно приближаться к нему ближе и ближе, но сам предел недостигаем.

Возможно, математически это связано со следующим обстоятельством. Дело в том, что на графике температура-энтропия или объем-давление точки, в которых происходит перескок, лежат на односвязной кривой, в то время как во всех примерах, приведенных ниже для фазового перехода нулевого рода, они принадлежат разным аналитическим кривым. Чисто математически и эмпирически это обстоятельство действительно создает больший барьер между метастабильным и равновесным состояниями. Хотя в рамках термодинамического предела та часть графика температура-энтропия, которая связывает метастабильное и равновесное состояния, неустойчива и не должна "ощущаться". Ответ нужно искать в асимптотике для статистической физики.

ПримеР 2. Автором объяснен эффект фонтанирования, открытый в 1938 году Алленом и Джонсом, когда по капилляру диаметром $10^{-4}$ см “текла" сверхтекучая жидкость (на самом деле она находилась на сверхтекучем уровне метастабильного состояния). На двухуровневой модели автором показано, что в точке, нагретой (светом) до температуры фазового перехода $\theta_{\text {c }}$, происходит обращение в бесконечность теплоемкости, скачок энтропии и свободной энергии до нижнего значения свободной энергии, т.е. до той точки, в которую приходит кривая из основного состояния, нагретого до температуры $\theta_{\text {c }}$. Это значит, что происходит фазовый переход, который 
естественно назвать фазовым переходом нулевого рода. Это явление легко было бы объяснить, если предположить, что сверхтекучесть - не термодинамическое состо-

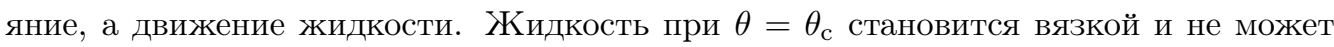
проникать в тонкий капилляр. Но дело в том, что это неверно, сверхтекучесть - это не движение, а состояние, и тогда это переход нулевого рода.

ПримеР 3. Рассмотрим подробнее пример, приведенный в п. 2.1.5. Будем медленно менять константу $q$. Отметим, что в термодинамике, учитывающей поле, имеются еще две термодинамические величины: напряженность поля и заряд. Таким образом, изменение константы $q$ есть изменение термодинамической переменной.

Покажем, что переход параметра $q$ через точку ноль приводит к фазовому переходу нулевого рода. Действительно, при $q=0$ возникает резонанс: собственные функции уже не сосредоточены в одной из впадин и вероятность (квадрат каждой собственной функции) одинаково распределена по обеим впадинам. Собственных значений становится "вдвое" больше, и распределение Гиббса меняется скачком. Именно здесь можно увидеть роль времени. Чтобы половина функции, убывающей вне первой впадины, “перекачалась" во вторую, должно пройти большое время. Однако формула Гиббса это не учитывает. Ждать этого перехода, может быть, придется долго, как и в предыдущем примере с толстым капилляром.

Если параметр $q$ становится отрицательным, то остаются все собственные функции как в одной, так и в другой впадине, и в формуле Гиббса рассматриваются все собственные значения, а не их подмножество.

2.2. Точно решаемая модель. Вначале мы рассмотрим одномерное уравнение Шредингера для одной частицы на окружности

$$
\widehat{H} \psi_{k}(x)=E \psi_{k}(x), \quad \psi_{k}(x-L)=\psi_{k}(x),
$$

где $\psi_{k}(x)$ - волновая функция, $x$ принимает значения на окружности, а $\widehat{H}$ - дифференциальный оператор вида

$$
\widehat{H}=\varepsilon\left(-i \hbar \frac{\partial}{\partial x}\right), \quad \varepsilon(z) \in C^{\infty},
$$

$\hbar$ - постоянная Планка, $\psi_{k}(x)=e^{i p_{k} x}, p_{k}=2 \pi \hbar k / L, k$ - произвольное целое число, соответствующие собственные значения равны

$$
E_{k}=\varepsilon\left(p_{k}\right)
$$

Перейдем от гамильтоновой функции $\varepsilon(p)$ к дискретной функции $\tilde{\varepsilon}(p)$, имеющей вид

$$
\tilde{\varepsilon}(p)=\varepsilon(n \Delta p), \quad \Delta p\left(n-\frac{1}{2}\right) \leqslant p<\Delta p\left(n+\frac{1}{2}\right),
$$

где $n$ - произвольное целое число, $\Delta p$ - некоторая положительная постоянная. Гамильтониан (2) при таком переходе изменяется соответствующим образом, будем 
обозначать его $\widehat{\widetilde{H}}$, собственные функции этого оператора совпадают с $\psi_{k}(x)$, а собственные значения (3) переходят в

$$
\widetilde{E}_{k}=\tilde{\varepsilon}\left(p_{k}\right) .
$$

Будем далее считать, что постоянная $\Delta p$ имеет вид

$$
\Delta p=\frac{2 \pi \hbar G}{L},
$$

где $G$ - натуральное число. В таком случае из (4) следует, что набор энергетических уровней $(5)$ является набором $G$-кратно вырожденных энергетических уровней

$$
\lambda_{n}=\widetilde{E}_{G n}=\varepsilon\left(p_{G n}\right) .
$$

Уравнение Шредингера для $N$ невзаимодействующих частиц имеет вид

$$
\widehat{H}_{N} \Psi\left(x_{1}, \ldots, x_{N}\right)=E \Psi\left(x_{1}, \ldots, x_{N}\right),
$$

где $\Psi\left(x_{1}, \ldots, x_{N}\right)$ - симметричная функция переменных $x_{1}, \ldots, x_{N}$ (бозоны), а гамильтониан $\widehat{H}_{N}$ выражается формулой

$$
\widehat{H}_{N}=\sum_{j=1}^{N} \widehat{\widetilde{H}}_{j}
$$

где $\widehat{\widetilde{H}}_{j}$ - гамильтониан частицы с номером $j$, который имеет вид

$$
\widehat{\widetilde{H}}_{j}=\tilde{\varepsilon}\left(-i \hbar \frac{\partial}{\partial x_{j}}\right) .
$$

Полная ортонормированная система симметричных собственных функций гамильтониана (9) имеет вид

$$
\Psi_{\{N\}}\left(x_{1}, \ldots, x_{N}\right)=\operatorname{Symm}_{x_{1}, \ldots, x_{N}} \psi_{\{N\}}\left(x_{1}, \ldots, x_{N}\right),
$$

где $\operatorname{Symm}_{x_{1}, \ldots, x_{N}}$ - оператор симметризации по переменным $x_{1}, \ldots, x_{N},\{N\}$ - набор целых неотрицательных чисел $N_{k}, k \in \mathbb{Z}$, удовлетворяющих условию

$$
\sum_{k=-\infty}^{\infty} N_{k}=N
$$

а функция $\psi_{\{N\}}\left(x_{1}, \ldots, x_{N}\right)$ равна

$$
\psi_{\{N\}}\left(x_{1}, \ldots, x_{N}\right)=\prod_{s=1}^{N} \psi_{k_{s}}\left(x_{s}\right),
$$

где индексы $k_{1}, \ldots, k_{N}$ выражаются через набор $\{N\}$ из условий

$$
\begin{array}{ll}
k_{s} \leqslant k_{s+1} & \text { для всех } \quad 1 \leqslant s \leqslant N-1, \\
\sum_{s=1}^{N} \delta_{k k_{s}}=N_{k} \quad \text { для всех } \quad k \in \mathbb{Z},
\end{array}
$$


здесь $\delta_{k k^{\prime}}$ - символ Кронекера. Собственные значения гамильтониана (9) равны

$$
E(\{N\})=\sum_{k=-\infty}^{\infty} \widetilde{E}_{k} N_{k}
$$

Рассмотрим взаимодействие между частицами следующего вида. Будем считать, что частицы взаимодействуют парами и оператор взаимодействия частиц с номерами $j$ и $k$ имеет вид

$$
\widehat{V}_{j k}=-\frac{V}{N} W\left(\widehat{\widetilde{H}}_{j}-\widehat{\widetilde{H}}_{k}\right)
$$

где $V>0$ - параметр величины взаимодействия, а функция $W(\xi)$ равна

$$
W(\xi)= \begin{cases}1, & |\xi|<d \\ 0, & |\xi| \geqslant d\end{cases}
$$

здесь $d>0$ - радиус взаимодействия по энергии. Оператор (17) соответствует взаимодействию, при котором пара частиц притягивается друг к другу и испускает квант энергии $-V / N$, если разность их энергий меньше $d$, а если разность энергий частиц больше $d$, то частицы не взаимодействуют. Гамильтониан системы $N$ частиц с взаимодействием (17) принимает вид

$$
\widehat{H}_{N}=\sum_{j=1}^{N} \widehat{\widetilde{H}}_{j}+\sum_{j=1}^{N} \sum_{k=j+1}^{N} \widehat{V}_{j k}
$$

В силу (16) суммы в выражении (18) коммутируют, поэтому набор собственных функций гамильтониана (18) совпадает с (11), также из (16) следует, что соответствующие собственные значения равны

$$
E(\{N\})=\sum_{k=-\infty}^{\infty} \widetilde{E}_{k} N_{k}-\frac{V}{2 N} \sum_{k=-\infty}^{\infty} \sum_{l=-\infty}^{\infty} W\left(\widetilde{E}_{k}-\widetilde{E}_{l}\right)\left(N_{k} N_{l}-\delta_{k l} N_{k}\right) .
$$

Далее будем считать, что радиус взаимодействия достаточно мал и для него выполняется условие

$$
d<\min _{n \neq m}\left|\lambda_{n}-\lambda_{m}\right|
$$

Набор энергетических уровней $\widetilde{E}_{k}, k \in \mathbb{Z}$, совпадает с $G$-кратно вырожденным набором уровней (7). Значит, в силу (20) набор энергетических уровней (19) рассматриваемой системы $N$ частиц может быть записан в виде

$$
\mathbf{E}(\{\tilde{N}\})=\sum_{n=-\infty}^{\infty} \lambda_{n} \widetilde{N}_{n}-\frac{V}{2 N} \sum_{n=-\infty}^{\infty} \tilde{N}_{n}\left(\tilde{N}_{n}-1\right),
$$

где уровень $\mathbf{E}(\{\tilde{N}\})$ имеет кратность

$$
\Gamma(\{\tilde{N}\})=\prod_{n=-\infty}^{\infty} \frac{\left(G+\widetilde{N}_{n}-1\right) !}{(G-1) ! \widetilde{N}_{n} !} .
$$


Здесь $\{\widetilde{N}\}$ обозначает набор целых неотрицательных чисел $\widetilde{N}_{n}, n \in \mathbb{Z}$, для которых выполняется условие

$$
\sum_{n=-\infty}^{\infty} \widetilde{N}_{n}=N
$$

Рассмотрим статистическую сумму для системы $N$ бозонов с гамильтонианом (18). Так как энергетические уровни и их кратности выражаются формулами (21) и (22) соответственно, то статистическая сумма при температуре $\theta$ принимает вид

$$
Z(\theta, N)=\sum_{\{\tilde{N}\}} \Gamma(\{\tilde{N}\}) \exp [-\mathbf{E}(\{\tilde{N}\}) \theta],
$$

суммирование здесь ведется по всем наборам $\{\tilde{N}\}$ с учетом условия (23).

Будем считать далее, что $G$ зависит от $N$, причем выполняется условие

$$
\lim _{N \rightarrow \infty} \frac{G}{N}=g>0
$$

Обозначим через $\widetilde{F}(\{\widetilde{N}\}, \theta)-$ функцию вида

$$
\widetilde{F}(\{\tilde{N}\}, \theta)=\mathbf{E}(\{\tilde{N}\})-\theta \ln (\Gamma(\{\tilde{N}\})),
$$

$\left\{\widetilde{N}^{0}\right\}$ - набор неотрицательных чисел $\widetilde{N}_{n}^{0}, n \in \mathbb{Z}$, для которых функция (26) минимальна при условии (23).

Рассмотрим теперь вопрос о нахождении минимального значения функции (26) при условии (23). В пределе при $N \rightarrow \infty$ и при выполненном условии (25) точка минимума имеет вид

$$
\widetilde{N}_{n}(\theta)=N\left(m_{n}(\theta)+o(1)\right),
$$

где $m_{n}(\theta), n \in \mathbb{Z}$, определяется из системы уравнений

$$
\lambda_{n}-V m_{n}+\theta \ln \left(\frac{m_{n}}{g+m_{n}}\right)=\mu(\theta), \quad n \in \mathbb{Z},
$$

а $\mu(\theta)$ находится из уравнения

$$
\sum_{n=-\infty}^{\infty} m_{n}=1
$$

Подстановка $(27)$ в (26) и использование при этом асимптотической формулы Стирлинга приводит к следующему равенству для удельной свободной энергии:

$$
\begin{aligned}
f(\theta) & \equiv \lim _{N \rightarrow \infty} f(\theta, N)=\lim _{N \rightarrow \infty} \frac{\tilde{F}\left(\left\{\tilde{N}^{0}\right\}, \theta\right)}{N}= \\
& =\sum_{n=-\infty}^{\infty}\left(\lambda_{n} m_{n}-\frac{V}{2} m_{n}^{2}\right)+\left(\theta m_{n} \ln \left(\frac{m_{n}}{g}\right)-\theta\left(g+m_{n}\right) \ln \left(1+\frac{m_{n}}{g}\right)\right),
\end{aligned}
$$

где для краткости у $m_{n}(\theta)$ опущен аргумент $\theta$. Введем обозначение

$$
\omega_{n}=\lambda_{n}-V m_{n} .
$$


ЗАмечАниЕ. Система уравнений (28) и (29) в обозначениях (31) принимает вид

$$
\begin{gathered}
\omega_{n}(\theta)=\lambda_{n}-V \frac{g}{e^{\left(\omega_{n}-\mu\right) / \theta}-1}, \quad n \in \mathbb{Z}, \\
\sum_{n=-\infty}^{\infty} \frac{g}{e^{\left(\omega_{n}-\mu\right) / \theta}-1}=1 .
\end{gathered}
$$

Система уравнений (32) точно совпадает с температурными уравнениями Хартри для системы $N$ бозонов с гамильтонианом (18).

Исследуем решения системы уравнений (28), (29). При $\theta=0$ система имеет множество решений, которое будем нумеровать целым числом $l$ :

$$
m_{n}^{(l)}=\delta_{l n}, \quad n, l \in \mathbb{Z} .
$$

Отберем из всех номеров $l$ такие, для которых выполняется условие

$$
\nu_{n l} \equiv \lambda_{n}-\lambda_{l}+V>0 \quad \text { для всех } n \neq l \text {. }
$$

Для этих номеров существуют решения системы уравнений (28), (29), которые при $\theta \rightarrow 0$ сходятся к (33). Асимптотика этих решений при $\theta \rightarrow 0$ имеет вид

$$
\begin{gathered}
m_{n}^{(l)} \sim g e^{-\nu_{n l} / \theta} \quad \forall n \neq l, \\
1-m_{l}^{(l)} \sim g \sum_{n \neq l} e^{-\nu_{n l} / \theta} .
\end{gathered}
$$

Итак, в зависимости от спектра $\lambda_{n}, n \in \mathbb{Z}$, при достаточно малых значениях температуры $\theta$ система уравнений (28), (29) имеет множество решений. Эти решения кроме точки глобального минимума содержат также точки локального минимума функции (26). Значения функции (26) в точках локального минимума равны свободной энергии метастабильных состояний. Рассмотрим функцию (26) при $\theta=0$. Она в этом случае совпадает с энергетическим спектром системы (21). Рассмотрим энергию системы в случае, когда почти все частицы находятся на энергетическом уровне $\lambda_{l}$. Это означает, что выполняются условия

$$
\tilde{N}_{n} \ll N \quad \forall n \neq l .
$$

Выражая $\widetilde{N}_{l}$ из равенства (23) и подставляя результат в (21), в силу (36) получим, что энергетический спектр системы в этой области имеет вид

$$
\mathbf{E}(\{\tilde{N}\}) \approx \lambda_{l} N-\frac{V N}{2}+\sum_{n \neq l}\left(\lambda_{n}-\lambda_{l}+V\right) \tilde{N}_{n}
$$

Гамильтониану (18) соответствуют уравнение Хартри и система уравнений в вариациях. Каждому $l \in \mathbb{Z}$ соответствует решение уравнения Хартри, описывающее состояние

$$
\widetilde{N}_{n}^{(l)}=N \delta_{n l}
$$

5 Теоретическая и математическая физика, т. 150, № 1, 2007 г. 
Кроме того, собственные значения системы уравнений в вариациях, отвечающей этому решению уравнения Хартри, совпадают с (34). Можно показать, что если собственные значения системы уравнений в вариациях для решения уравнения Хартри действительны и неотрицательны, то такое решение соответствует основному или метастабильному состоянию системы. Это означает, что всем $l$, для которых выполняется (34), при $\theta=0$ соответствуют метастабильные состояния системы бозонов. По аналогии со случаем нулевой температуры решениям системы уравнений (28), (29) при $\theta \neq 0$ соответствует температурное метастабильное состояние, если точка (27) является точкой локального минимума. Заметим теперь, что при очень больших температурах у системы уравнений (28), (29) есть только одно решение, соответствующее глобальному минимуму функции (26). Асимптотика этого решения при $\theta \rightarrow \infty$ имеет вид

$$
n_{m}(\theta) \sim g \frac{e^{-\lambda_{m} / \theta}}{\sum_{l=-\infty}^{\infty} e^{-\lambda_{l} / \theta}} .
$$

Единственность решения при больших температурах означает, что все метастабильные состояния с увеличением температуры исчезают. Температура, при которой исчезает метастабильное состояние, является критической для этого состояния.

Исследуем вопрос о поведении энтропии и теплоемкости метастабильных состояний при приближении к критической температуре. Рассмотрим метастабильное состояние, которому соответствует решение системы уравнений (28), (29), сходящееся при $\theta \rightarrow 0$ к (33) для некоторого $l$, для которого выполняется (34). Будем считать далее, что функция $\varepsilon(p)$ такова, что $\varepsilon(0)<\varepsilon(p)$ при $p \neq 0$. Тогда согласно (7) $\lambda_{0}<\lambda_{l}$ при $l \neq 0$. Поэтому решение системы уравнений (28), (29), сходящееся при $\theta \rightarrow 0$ к $(33)$ для $l=0$, соответствует основному температурному состоянию системы $N$ бозонов. Кроме того, условие (34) становится эквивалентно условию $\lambda_{l}-\lambda_{0}<V$.

Будем считать, что для $l \neq 0$ выполнено это неравенство. Условие того, что соответствующее решение $m_{n}^{(l)}(\theta)$ системы уравнений $(28),(29)$ определяет по формуле (27) точку локального минимума функции (26) при условии (23), имеет вид системы неравенств:

$$
\begin{aligned}
\alpha_{n}^{(l)}(\theta) \equiv- & V+\frac{\theta g}{m_{n}^{(l)}(\theta)\left(g+m_{n}^{(l)}(\theta)\right)}>0 \quad \forall n \neq l \\
\alpha_{l}^{(l)}(\theta) \equiv- & V+\frac{\theta g}{m_{l}^{(l)}(\theta)\left(g+m_{l}^{(l)}(\theta)\right)}<0, \\
& -\sum_{n \neq l} \frac{\alpha_{l}^{(l)}(\theta)}{\alpha_{n}^{(l)}(\theta)}<1 .
\end{aligned}
$$

Заметим, что эти неравенства выполняются для асимпотики (35) при $\theta \rightarrow 0$. Неравенства (40) получаются из условия положительности второй вариации функции (26), вариация берется при условии (23).

Метастабильное состояние исчезает при температуре, при которой последнее неравенство из (40) становится равенством. Будем обозначать эту критическую температуру $\theta_{\mathrm{c}}^{(l)}$. Из (40) и (28), (29) при $\theta<\theta_{\mathrm{c}}^{(l)}$ следует, что $m_{n}^{(l)}(\theta)$ является растущей 
функцией переменной $\theta$ при $n \neq l$, а $m_{l}^{(l)}(\theta)$ является убывающей функцией $\theta$. Кроме того, получается, что $m_{l}^{(l)}(\theta)>m_{n}^{(l)}(\theta)>m_{n^{\prime}}^{(l)}(\theta)$, если $\lambda_{n}<\lambda_{n^{\prime}}$ и $n, n^{\prime} \neq l$.

Из (30) следует выражение для удельной энтропии метастабильного состояния в пределе при $N \rightarrow \infty$ :

$$
s^{(l)}(\theta)=\sum_{n=-\infty}^{\infty}\left(\left(g+m_{n}^{(l)}\right) \ln \left(1+\frac{m_{n}^{(l)}}{g}\right)-m_{n}^{(l)} \ln \left(\frac{m_{n}^{(l)}}{g}\right)\right),
$$

где для краткости у $m_{n}^{(l)}(\theta)$ опущен аргумент $\theta$. Дифференцируя $(41)$, получим

$$
\frac{\partial s}{\partial \theta}=\sum_{n \neq l} \frac{\partial m_{n}^{(l)}}{\partial \theta} \ln \left(\frac{g+m_{n}^{(l)}}{m_{n}^{(l)}} \frac{m_{l}^{(l)}}{g+m_{l}^{(l)}}\right)>0 .
$$

Последнее неравенство вытекает из свойств функции $m_{n}^{(l)}(\theta)$. Поскольку при критической температуре последнее из неравенств (40) превращается в равенство, можно показать, что при $\theta \rightarrow \theta_{\mathrm{c}}^{(l)}-0$ решения уравнений $(28),(29)$ ведут себя следующим образом:

$$
m_{n}^{(l)}(\theta)-m_{n}^{(l)}\left(\theta_{\mathrm{c}}^{(l)}\right) \approx \frac{C^{(l)}}{\alpha_{n}^{(l)}\left(\theta_{\mathrm{c}}^{(l)}\right)} \sqrt{\theta_{\mathrm{c}}^{(l)}-\theta} \quad \forall n \in \mathbb{Z},
$$

где $C^{(l)}$ - отрицательное число. Подстановка (43) в (41) показывает, что производная удельной энтропии по температуре (эта величина равна теплоемкости, деленной на температуру) при $\theta \rightarrow \theta_{\mathrm{c}}^{(l)}-0$ стремится к бесконечности как $1 / \sqrt{\theta_{\mathrm{c}}^{(l)}-\theta}$. Это значит, что проецирование лагранжева многообразия, соответствующего метастабильному состоянию, на ось $\theta$ становится неоднозначным в окрестности критической температуры. Производная температуры по энтропии при приближении к критической температуре обращается в нуль, поэтому, а также в силу (42), как это уже было отмечено выше, лагранжево многообразие однозначно проецируется на ось $s$. Отметим, что из свойств функции $m_{n}^{(l)}(\theta)$ следует, что при $\theta<\theta_{\mathrm{c}}^{(l)}$ выполняются неравенства

$$
\begin{array}{cc}
m_{0}^{(l)}(\theta)<m_{n}^{(l)}(\theta) & \forall n \neq 0, l, \\
\alpha_{0}^{(l)}(\theta)<\alpha_{n}^{(l)}(\theta) & \forall n \neq 0, l .
\end{array}
$$

Эти неравенства означают, что потенциальный барьер между энергетическими уровнями $\lambda_{l}$ и $\lambda_{0}$ меньше, чем энергетический барьер между $\lambda_{l}$ и $\lambda_{n}$ при $n \neq 0, l$. Это значит, что рассматриваемая система бозонов, находящаяся в метастабильном состоянии с номером $l$, при достижении критической температуры скачком изменяет свое состояние и переходит в основное температурное состояние. Это фазовый переход нулевого рода, поскольку скачком изменяются не только энтропия и теплоемкость, но также и свободная энергия.

Когда величина $\min _{n \neq 0}\left|\lambda_{n}-\lambda_{0}\right|=\delta$ мала, разность свободных энергий при фазовом переходе нулевого рода из самого нижнего метастабильного состояния в основное состояние также является малой, а теплоемкость при таком переходе имеет особенность. Асимптотика статистической суммы около критической точки дается 
асимптотикой канонического оператора в окрестности фокальной точки. Эта асимптотика имеет вид функции типа Эйри от мнимого аргумента и имеет особенность при $N \rightarrow \infty$. Можно подобрать параметры $\delta \ll 1, N \gg 1$ и $L \gg 1$ так, что вид точки фазового перехода будет в точности совпадать с $\lambda$-точкой.

\section{3. О ЧАСТОТЕ ФАЗОВЫХ ПЕРЕХОДОВ НУЛЕВОГО РОДА}

Упорядочим по величине последовательность случайных чисел $\lambda_{1}, \ldots, \lambda_{s}$, как это было сделано в работе [14]. Среди чисел $\lambda_{1}, \ldots, \lambda_{s}$ могут быть и равные. В этом случае их объединим, складывая при этом отвечающие им "вероятности", т.е. отношение числа выпадения значения $\lambda_{i}$ к общему числу испытаний. Число равных $\lambda_{i}: \lambda_{i}=\lambda_{i+1}=\cdots=\lambda_{i+k}$ будем называть кратностью $q_{i}$ значения $\lambda_{i}$. $\mathrm{B}$ нашем рассмотрении к бесконечности стремятся как число испытаний $N$, так и $s$.

Пусть $N_{i}$ - число выпадений значения $\lambda_{i}: \lambda_{i}<\lambda_{i+1}$, тогда математическое ожидание

$$
M=\sum_{i=1}^{s} \frac{N_{i}}{N} \lambda_{i} .
$$

Кумулятивная вероятность $\mathcal{P}_{k}$ есть сумма первых $k$ вероятностей в последовательности $\lambda_{i}: \mathcal{P}_{k}=\frac{1}{N} \sum_{i=1}^{k} N_{i}$, где $k<s$. Обозначим $N \mathcal{P}_{k}=B_{k}$.

Если все варианты, для которых

$$
\begin{gathered}
\sum_{i=1}^{s} N_{i}=N, \\
\sum_{i=1}^{s} N_{i} \lambda_{i} \leqslant E, \quad E=M N \leqslant N, \bar{\lambda},
\end{gathered}
$$

где $\bar{\lambda}=\left(\sum_{i=1}^{s} q_{i} \lambda_{i}\right) / Q, Q=\sum_{i=1}^{s} q_{i}$, равноценны (равновероятны), то большинство вариантов будут скапливаться около следующей зависимости кумулятивной вероятности [15]-[17]:

$$
B_{l}\left\{N_{i}\right\}=\sum_{i=1}^{l} N_{i}=\sum_{i=1}^{l} \frac{q_{i}}{e^{\beta^{\prime} \lambda_{i}-\nu^{\prime}}-1}
$$

где $\beta^{\prime}$ и $\nu^{\prime}$ определяются из условий

$$
\begin{gathered}
B_{s}=N, \quad \text { если } N \rightarrow \infty \text { и } s \rightarrow \infty, \\
\sum_{i=1}^{s} \frac{q_{i} \lambda_{i}}{e^{\beta^{\prime} \lambda_{i}-\nu^{\prime}}-1}=E .
\end{gathered}
$$

Предположим, что $a_{1} N \leqslant Q \leqslant a_{2} N$, где $a_{1}, a_{2}$ - константы. Введем обозначения: $\mathcal{M}$ - множество всех наборов $\left\{N_{i}\right\}$, удовлетворяющих условиям (46), $\mathcal{N}\{\mathcal{M}\}$ - число элементов множества $\mathcal{M}$. 
ТЕОремА. Пусть все варианты наборов $\left\{N_{i}\right\}$, для которых выполняются условия (46), равновероятны. Тогда число вариантов $\mathcal{N}$ наборов $\left\{N_{i}\right\}$, для которых выполняется дополнительное соотношение

$$
\left|\sum_{i=1}^{l} N_{i}-\sum_{i=1}^{l} \frac{q_{i}}{e^{\beta^{\prime} \lambda_{i}-\nu^{\prime}}-1}\right| \geqslant N^{(3 / 4+\varepsilon)},
$$

менъше, чем $c_{1} \mathcal{N}\{\mathcal{M}\} / N^{m}$, где $c_{1}$ и $m$-любые числа, $\sum_{i=1}^{l} q_{i} \geqslant \varepsilon Q, \varepsilon-$ сколь угодно малое число.

ДокАЗАТЕЛЬСТво. Пусть $\mathcal{A}$ - подмножество $\mathcal{M}$, удовлетворяющее условию

$$
\begin{gathered}
\left|\sum_{i=l+1}^{s} N_{i}-\sum_{i=l+1}^{s} \frac{q_{i}}{e^{\beta \lambda_{i}-\nu}-1}\right| \leqslant \Delta, \\
\left|\sum_{i=1}^{l} N_{i}-\sum_{i=1}^{l} \frac{q_{i}}{e^{\beta^{\prime} \lambda_{i}-\nu^{\prime}}-1}\right| \leqslant \Delta,
\end{gathered}
$$

где $\Delta, \beta, \nu$ - некоторые действительные числа, не зависящие от $l$. Обозначим

$$
\begin{gathered}
\left|\sum_{i=l+1}^{s} N_{i}-\sum_{i=l+1}^{s} \frac{q_{i}}{e^{\beta \lambda_{i}-\nu}-1}\right|=S_{s-l}, \\
\left|\sum_{i=1}^{l} N_{i}-\sum_{i=1}^{l} \frac{q_{i}}{e^{\beta^{\prime} \lambda_{i}-\nu^{\prime}}-1}\right|=S_{l} .
\end{gathered}
$$

Очевидно, что если $\left\{N_{i}\right\}$ - множество всех наборов целых чисел, то

$$
\mathcal{N}\{\mathcal{M} \backslash \mathcal{A}\}=\sum_{\left\{N_{i}\right\}}\left(\Theta\left(E-\sum_{i=1}^{s} N_{i} \lambda_{i}\right) \delta_{\left(\sum_{i=1}^{s} N_{i}\right), N} \Theta\left(S_{l}-\Delta\right)\right) \Theta\left(S_{s-l}-\Delta\right)
$$

где первое суммирование ведется по всем целым $N_{i}, \Theta(\lambda)$ - функция Хевисайда, а $\delta_{k_{1}, k_{2}}$ - символ Кронекера. Используем интегральные представления

$$
\begin{aligned}
\delta_{N N^{\prime}} & =\frac{e^{-\nu N}}{2 \pi} \int_{-\pi}^{\pi} d \varphi e^{-i N \varphi} e^{\nu N^{\prime}} e^{i N^{\prime} \varphi}, \\
\Theta(y) & =\frac{1}{2 \pi i} \int_{-\infty}^{\infty} d \lambda \frac{1}{\lambda-i} e^{\beta y(1+i \lambda)} .
\end{aligned}
$$

Прибегнем к стандартной регуляризации. Заменим первую $\Theta$-функцию Хевисайда в формуле (51) на непрерывную функцию

$$
\begin{aligned}
& \Theta_{\alpha}(y)=\left\{\begin{array}{lll}
0, & \alpha>1, & y<0, \\
1-e^{\beta y(1-\alpha)}, & \alpha>1, & y \geqslant 0,
\end{array}\right. \\
& \Theta_{\alpha}(y)= \begin{cases}e^{\beta y(1-\alpha)}, & \alpha<0, \quad y<0, \\
1, & \alpha<0, \quad y \geqslant 0,\end{cases}
\end{aligned}
$$


где $\alpha \in(-\infty, 0) \cup(1, \infty)$ - параметр. Отсюда

$$
\Theta_{\alpha}(y)=\frac{1}{2 \pi i} \int_{-\infty}^{\infty} d x e^{\beta y(1+i x)}\left(\frac{1}{x-i}-\frac{1}{x-\alpha i}\right) .
$$

Если $\alpha^{\prime}>1$, то $\Theta(y) \leqslant \Theta_{\alpha}(y)$.

Пусть $\nu<0$. Подставляя (52) в (51), меняя местами интегрирование и суммирование и затем переходя к пределу при $\alpha \rightarrow \infty$, получаем оценку

$$
\begin{aligned}
\mathcal{N}\{\mathcal{M} \backslash \mathcal{A}\} & \leqslant \mid \frac{e^{-\nu N+\beta E}}{i(2 \pi)^{2}} \int_{-\pi}^{\pi} d \varphi e^{-i N \varphi} \times \\
& \times \sum_{\left\{N_{j}\right\}} \exp \left\{-\beta \sum_{j=1}^{s} N_{j} \lambda_{j}+(i \varphi+\nu) \sum_{j=1}^{s} N_{j}\right\} \Theta\left(S_{l}-\Delta\right) \Theta\left(S_{s-l}-\Delta\right) \mid,
\end{aligned}
$$

где $\beta$ и $\nu$ - такие действительные параметры, для которых ряд сходится. Пронеся модуль под интеграл и затем под знак суммы и интегрируя по $\varphi$, получим

$$
\mathcal{N}\{\mathcal{M} \backslash \mathcal{A}\} \leqslant \frac{e^{-\nu N+\beta E}}{2 \pi} \sum_{\left\{N_{i}\right\}} \exp \left\{-\beta \sum_{i=1}^{s} N_{i} \lambda_{i}+\nu \sum_{i=1}^{s} N_{i}\right\} \Theta\left(S_{l}-\Delta\right) \Theta\left(S_{s-l}-\Delta\right)
$$

Обозначим

$$
Z(\beta, N)=\sum_{\left\{N_{i}\right\}} \exp \left\{-\beta \sum_{i=1}^{s} N_{i} \lambda_{i}\right\}
$$

где здесь уже сумма берется по всем $N_{i}$ таким, что $\sum_{i=1}^{s} N_{i}=N$,

$$
\begin{gathered}
\zeta_{l}(\nu, \beta)=\prod_{i=1}^{l} \xi_{i}(\nu, \beta), \quad \zeta_{s-l}(\nu, \beta)=\prod_{i=l+1}^{s} \xi_{i}(\nu, \beta), \\
\xi_{i}(\nu, \beta)=\frac{1}{\left(1-e^{\nu-\beta \lambda_{i}}\right)^{q_{i}}}, \quad i=1, \ldots, l .
\end{gathered}
$$

Из неравенства для гиперболического косинуса

$$
\operatorname{ch} x_{1} \operatorname{ch} x_{2}=\operatorname{ch}\left(x_{1}+x_{2}\right)+\operatorname{ch}\left(x_{1}-x_{2}\right)>\frac{e^{\delta}}{2},
$$

справедливого при $\left|x_{1}\right| \geqslant \delta,\left|x_{2}\right| \geqslant \delta$, следует, что для всех положительных $c$ и $\Delta$ выполняется неравенство

$$
\Theta\left(S_{s-l}-\Delta\right) \Theta\left(S_{l}-\Delta\right) \leqslant e^{-c \Delta} \operatorname{ch}\left(c \sum_{i=1}^{l} N_{i}-c \phi_{l}\right) \operatorname{ch}\left(c \sum_{i=l+1}^{s} N_{i}-c \bar{\phi}_{s-l}\right)
$$

где

$$
\phi_{l}=\sum_{i=1}^{l} \frac{q_{i}}{e^{\beta^{\prime} \lambda_{i}-\nu^{\prime}}-1}, \quad \bar{\phi}_{s-l}=\sum_{i=l+1}^{s} \frac{q_{i}}{e^{\beta \lambda_{i}-\nu}-1} .
$$


Получаем

$$
\begin{aligned}
\mathcal{N}\{\mathcal{M} \backslash \mathcal{A}\} \leqslant & e^{-c \Delta} e^{\beta E-\nu N} \sum_{\left\{N_{i}\right\}} \exp \left\{-\beta \sum_{i=1}^{l} N_{i} \lambda_{i}+\nu \sum_{i=1}^{l} N_{i}\right\} \operatorname{ch}\left(\sum_{i=1}^{l} c N_{i}-c \phi\right) \times \\
& \times \exp \left\{-\beta \sum_{i=l+1}^{s} N_{i} \lambda_{i}+\nu \sum_{i=l+1}^{s} N_{i}\right\} \operatorname{ch}\left(\sum_{i=l+1}^{s} c N_{i}-c \bar{\phi}\right)= \\
= & e^{\beta E} e^{-c \Delta}\left(\zeta_{l}(\nu-c, \beta) e^{-c \phi_{l}}+\zeta_{l}(\nu+c, \beta) e^{c \phi_{l}}\right) \times \\
& \times\left(\zeta_{s-l}(\nu-c, \beta) e^{-c \bar{\phi}_{s-l}}+\zeta_{s-l}(\nu+c, \beta) e^{c \bar{\phi}_{s-l}}\right)
\end{aligned}
$$

Используем равенства

$$
\left.\frac{\partial}{\partial \nu} \ln \zeta_{l}\right|_{\beta=\beta^{\prime}, \nu=\nu^{\prime}} \equiv \phi_{l},\left.\quad \frac{\partial}{\partial \nu} \ln \zeta_{s-l}\right|_{\beta=\beta^{\prime}, \nu=\nu^{\prime}} \equiv \bar{\phi}_{s-l}
$$

и разложение $\zeta_{l}(\nu \pm c, \beta)$ по формуле Тейлора. Существует $\gamma<1$ такое, что

$$
\ln \left(\zeta_{l}(\nu \pm c, \beta)\right)=\ln \zeta_{l}(\nu, \beta) \pm c\left(\ln \zeta_{l}\right)_{\nu}^{\prime}(\nu, \beta)+\frac{c^{2}}{2}\left(\ln \zeta_{l}\right)_{\nu}^{\prime \prime}(\nu \pm \gamma c, \beta)
$$

Подставляя это разложение и используя формулу (59), получим, что $\phi_{\nu, \beta}$ сокращается.

Другое представление формулы Тейлора дает

$$
\begin{aligned}
\ln \left(\zeta_{l}(\nu+c, \beta)\right)=\ln \left(\zeta_{l}(\beta, \nu)\right)+\frac{c}{\beta} \frac{\partial}{\partial \nu} \ln \left(\zeta_{l}(\beta, \nu)\right)+ \\
\quad+\int_{\nu}^{\nu+c / \beta} d \nu^{\prime}\left(\nu+\frac{c}{\beta}-\nu^{\prime}\right) \frac{\partial^{2}}{\partial \nu^{\prime 2}} \ln \left(\zeta_{l}\left(\beta, \nu^{\prime}\right)\right) .
\end{aligned}
$$

Аналогичное выражение можно получить для $\zeta_{s-l}$.

Из явного вида функции $\zeta_{l}(\beta, \nu)$ имеем

$$
\frac{\partial^{2}}{\partial \nu^{2}} \ln \left(\zeta_{l}(\beta, \nu)\right)=\beta^{2} \sum_{i=1}^{l} \frac{g_{i} e^{-\beta\left(\lambda_{i}+\nu\right)}}{\left(e^{-\beta\left(\lambda_{i}+\nu\right)}-1\right)^{2}} \leqslant \beta^{2} Q d,
$$

где $d$ выражается формулой

$$
d=\frac{e^{-\beta\left(\lambda_{1}+\nu\right)}}{\left(e^{-\beta\left(\lambda_{1}+\nu\right)}-1\right)^{2}}
$$

Та же оценка имеет место для $\zeta_{s-l}$.

Учитывая, что $\zeta_{l} \zeta_{s-l}=\zeta_{s}$, получим оценку при $\beta=\beta^{\prime}, \nu=\nu^{\prime}$ :

$$
\mathcal{N}\{\mathcal{M} \backslash \mathcal{A}\} \leqslant \zeta_{s}\left(\beta^{\prime}, \nu^{\prime}\right) e^{-c \Delta+c^{2} \beta^{2} Q d / 2} e^{E \beta^{\prime}-\nu^{\prime} N} .
$$

Теперь выразим $\zeta_{s}\left(\nu^{\prime}, \beta^{\prime}\right)$ через $Z(\beta, N)$. Для этого докажем следующую лемму. 
Лемма 1. При условии (48) асимптотика интеграла

$$
Z(\beta, N)=\frac{e^{-\nu N}}{2 \pi} \int_{-\pi}^{\pi} d \alpha e^{-i N \alpha} \zeta_{s}(\beta, \nu+i \alpha)
$$

имеет вид

$$
Z(\beta, N)=C e^{-\nu N} \frac{\zeta_{s}(\beta, \nu)}{\left|\left(\partial^{2} \ln \zeta_{s}(\beta, \nu)\right) /\left(\partial^{2} \nu\right)\right|}\left(1+O\left(\frac{1}{N}\right)\right)
$$

где $C$ - константа.

ДокАЗАТЕЛЬСтво леммы 1 следует непосредственно из метода перевала и неравенств

$$
\left|\xi_{i}(\nu+i \alpha, \beta)\right|<\xi_{i}(\nu, \beta), \quad\left|\zeta_{s}(\nu+i \alpha, \beta)\right|<\zeta_{s}(\nu, \beta),
$$

которые выполняются в силу того, что $e^{\nu-\beta \lambda_{i}}<1$ для всех $\alpha \neq 2 \pi n$, где $n$ - целое число. Из них следует, что точка перевала интеграла (63) есть $\alpha=0$ [18].

Приведем другое доказательство. Имеем

$$
Z(\beta, N)=\frac{e^{-\nu N}}{2 \pi} \int_{-\pi}^{\pi} e^{-i N \alpha} \zeta_{s}(\beta, \nu+i \alpha) d \alpha=\frac{e^{-\nu N}}{2 \pi} \int_{-\pi}^{\pi} e^{N S(\alpha, N)} d \alpha,
$$

где

$$
S(\alpha, N)=-i \alpha+\ln \zeta_{s}(\beta, \nu+i \alpha)=-i \alpha-\sum_{i=1}^{s} q_{i} \ln \left(1-e^{\nu+i \alpha-\beta \lambda_{i}}\right) .
$$

Здесь функция $S$ зависит от $N$, поскольку от $N$ зависят $s, \lambda_{i}$ и $\nu$; последняя выбирается таким образом, чтобы точка $\alpha=0$ была стационарной точкой фазы $S$, т.е. из условия

$$
N=\sum_{i=1}^{s} \frac{q_{i}}{e^{\beta \lambda_{i}-\nu}-1} .
$$

Будем считать, что $a_{1} N \leqslant s \leqslant a_{2} N, a_{1}, a_{2}$ - константы и, кроме того, $0 \leqslant \lambda_{i} \leqslant B$, $B=$ const, $i=1,2, \ldots, s$. При выполнении этих условий в некотором интервале $\beta \in\left[0, \beta_{0}\right]$ значений обратной температуры все производные фазы ограничены, стационарная точка невырождена, и вещественная часть фазы вне окрестности нуля строго меньше ее значения в нуле минус некоторое положительное число. Поэтому при вычислении асимптотики интеграла можно заменить отрезок интегрирования $[-\pi, \pi]$ отрезком $[-\varepsilon, \varepsilon]$. Сделаем в этом интеграле замену переменной

$$
z=\sqrt{S(0, N)-S(\alpha, N)}
$$

Она голоморфна в круге $|\alpha| \leqslant \varepsilon$ в комплексной $\alpha$-плоскости и имеет голоморфную обратную, если $\varepsilon$ достаточно мало. В результате получаем

$$
\int_{-\varepsilon}^{\varepsilon} e^{N S(\alpha, N)} d \alpha=e^{N S(0, N)} \int_{\gamma} e^{-N z^{2}} f(z) d z
$$


где контур $\gamma$ в комплексной $z$-плоскости получается заменой (68) из отрезка $[-\varepsilon, \varepsilon]$, а

$$
f(z)=\left.\left(\frac{\partial \sqrt{S(0, N)-S(\alpha, N)}}{\partial \alpha}\right)^{-1}\right|_{\alpha=\alpha(z)} .
$$

При малом $\varepsilon$ контур $\gamma$ целиком лежит внутри двойного сектора $\operatorname{Re} z^{2}>c(\operatorname{Re} z)^{2}$ для некоторого $c>0$, поэтому его можно "сдвинуть" на вещественную ось, не меняя интеграла с точностью до экспоненциально малых по $N$ членов. Итак, с указанной точностью имеем

$$
Z(\beta, N)=\frac{e^{-\nu N}}{2 \pi} \int_{-\varepsilon}^{\varepsilon} e^{-N z^{2}} f(z) d z .
$$

Теперь, поскольку переменная $z$ стала вещественной, функцию $f(z)$ можно считать финитной (изменив ее вне отрезка интегрирования), распространить интеграл на всю ось (что снова даст экспоненциально малую ошибку) и затем вычислить асимптотическое разложение интеграла, разлагая подынтегральную функцию в ряд Тейлора по z с остаточным членом. Это обосновывает применение метода стационарной фазы к указанному интегралу для данного случая.

Лемма 2. Величина

$$
\frac{1}{\mathcal{N}(\mathcal{M})} \sum_{\left\{N_{i}\right\}} \exp \left(-\beta \sum_{i=1}^{s} N_{i} \lambda_{i}\right),
$$

где $\sum_{i=1}^{s} N_{i}=N, \lambda_{i} N_{i} \leqslant E-N^{1 / 2+\varepsilon}$, стремится $\kappa$ нулю быстрее $N^{-k}$ для любого $k, \varepsilon>0$.

ДокАЗАтЕЛЬСтво леммы 2. Рассмотрим точку минимума по $\beta$ правой части (58) при $\nu(\beta, N)$, удовлетворяющем условию

$$
\sum \frac{q_{i}}{e^{\beta \lambda_{i}-\nu(\beta, N)}-1}=N
$$

Нетрудно убедиться, что она удовлетворяет условиям (48), (49). Предположим, что условие леммы не выполняется. Тогда при $\sum N_{i}=N, \sum \lambda_{i} N_{i} \geqslant E-N^{1 / 2+\varepsilon}$

$$
e^{\beta E} \sum_{\left\{N_{i}\right\}} \exp \left(-\beta \sum_{i=1}^{s} N_{i} \lambda_{i}\right) \geqslant e^{\left(N^{1 / 2}+\varepsilon\right) \beta},
$$

и очевидно, что $\beta \ll 1 / \sqrt{N}$ дает минимум правой части (58) при выполнении леммы 1 , что противоречит тому, что минимум по $\beta$ правой части (58) равен $\beta^{\prime}$ (см. (62)).

Заметим, что по той же схеме Коваль [19] получил и прямое доказательство этой леммы.

Положим в формуле (62) после подстановки (64) $c=\Delta / N^{1+\alpha}$, тогда нетрудно видеть, что при $\Delta=N^{3 / 4+\varepsilon}$ отношение

$$
\frac{\mathcal{N}(\mathcal{M} \backslash \mathcal{A})}{\mathcal{N}(\mathcal{M})} \approx \frac{1}{N^{m}}
$$

где $m$ - любое целое число. Теорема доказана. 
Мы доказываем кумулятивную формулу, в которой плотности по виду совпадают с распределением Бозе-Эйнштейна. Отличие только в том, что в формуле вместо набора $\lambda_{n}$ случайных величин или собственных значений оператора Гамильтона стоят некоторые средние их значения по ячейкам. В силу доказанной теоремы $\varepsilon_{i}-$ средние значения энергии $\lambda_{k}$ в $i$-й ячейке - являются нелинейными средними по Колмогорову [16].

Перейдем к понятию размерности решетки. Рассмотрим прямую, плоскость и трех мерное пространство. На прямой мы отложим точки $i=0,1,2, \ldots$, на осях координат плоскости $(x, y)$ точки $x=i=0,1,2, \ldots, y=j=0,1,2, \ldots$. Сопоставим этому множеству точек $(i, j)$ точки на прямой натуральный ряд $l=1,2 \ldots$ с точностью до квантовой постоянной решетки $\chi$. Согласно определению Громова [20] асимптотическая (топологическая) размерность этой решетки равна двум. Сопоставим каждой точке пару точек $i$ и $j$ по правилу $i+j=l$. Число таких точек $n_{l}$ равно $l+1$. Если еще положить на оси $z=k=0,1,2, \ldots$, т.е. положить $i+j+k=l$, то в этом случае число точек $q_{l}$ будет равно $q_{l}=(l+1)(l+2) / 2$.

Если положить в формуле (47) $\lambda_{i}=l$, то в трехмерном случае каждому $i$ будет соответствовать $(l+1)(l+2) / 2$ равных между собой значений $x_{l}=l$ (это кратности или "вырожденности" спектра осциллятора). Формула (47) в этом частном случае будет выглядеть следующим образом:

$$
\begin{aligned}
N_{l} & =\text { const } \cdot \sum_{i=0}^{l} \frac{(i+1)(i+2)}{2\left(e^{\beta i-\nu}-1\right)}, \\
\Delta N_{i} & =\text { const } \cdot \frac{(i+1)(i+2)}{2\left(e^{\beta i-\nu}-1\right)} \Delta_{i}, \quad \Delta_{i}=1, \\
\Delta E_{i} & =\text { const } \cdot \frac{i(i+1)(i+2)}{2\left(e^{\beta i-\nu}-1\right)} \Delta_{i}
\end{aligned}
$$

при больших $i, \Delta_{i} / i \rightarrow 0$,

$$
d E=\text { const } \cdot \frac{\omega^{3} d \omega}{e^{\beta \omega}-1}, \quad \beta=\frac{h}{T}
$$

(ср. формулу (60.4) в [21]). Тем самым мы несколько уточняем знаменитую формулу Планка для излучения черного тела.

Нетрудно проверить для $D$-мерного случая, что последовательность весов (кратностей) числа вариантов $i=\sum_{k=1}^{D} m_{k}$, где $m_{k}$ - любые натуральные числа, имеет вид биномиального коэффициента

$$
q_{i}(D)=\text { const } \cdot \frac{(i+D-1) !}{i ! D !},
$$

где константа зависит от $D$.

Таким образом, формула (47) для любого $D$ имеет вид

$$
N_{l}=\text { const } \cdot \sum_{i=1}^{l} \frac{q_{i}(D)}{e^{\beta i}-1} .
$$


Для натурального ряда мы имеем последовательность весов $q_{i}$ (или просто вес) вида (75).

Нетрудно продолжить и наш весовой ряд на произвольный случай, заменив факториалы на Г-функции, при этом положим $D$ отрицательным. Это отрицательная топологическая размерность (дырочная размерность) квантованного пространства (решетки).

Если $D>1$, то в спектре осциллятора происходит сгущение при $i \rightarrow \infty$, при возникновении малого возмущения кратности расщепляются, т.е. с возрастанием $i$ спектр становится все гуще. Отрицательное значение $D$ при $i \rightarrow \infty$ означает сильное разрежение спектра (константа в формуле (76) должна быть достаточно большой).

При отрицательном целом значении $D$ члены $i=1, \ldots,-D$ обращаются в бесконечность. Это значит, что на эксперименте они очень велики. Это позволяет определить отрицательную размерность решетки, отвечающую данной задаче. Отметим, что имеет место новый конденсат, который возможен при малых $\beta$.

ПримеР. Частотный словарь составляется из текстов определенного массива. Каждому слову словаря сопоставлено число его встречаемости в исходном массиве текстов. Будем обозначать это число $\omega_{i}$, а число слов, отвечающих этому числу встречаемости, $-n_{i}$. Сумма всех $n_{i}$ равна объему всего словаря, а сумма произведений $\omega_{i} n_{i}$ - объему всего массива текстов, на основе которого составлен словарь.

Слова, которые встречаются в массиве текстов один раз, составляют примерно треть всего частотного словаря, количество слов которого равно $N$, т.е. $N / 3$, а это означает, что при $N \rightarrow \infty$ это конденсат. В силу вышесказанного для словаря $D=-1$. Значит, при $\beta \ll 1, \nu \sim 1$

$$
N_{l}=\mathrm{const} \cdot \sum_{i=2}^{l} \frac{1}{i(i-1)\left(e^{\beta i-\nu}-1\right)} \sim \mathrm{const} \cdot \int^{\omega} \frac{d \omega}{\alpha \omega(\alpha \omega-1)\left(e^{\beta \alpha \omega-\nu}-1\right)}
$$

при $\omega=l, \alpha-$ постоянная масштабирования.

Для частоты "японских свечей" для 30 акций на фондовой бирже (см. [22]) размерность равна нулю. Так что размерность частоты выбросов надо подбирать из эксперимента. Действительно, ведь о размерности пространства, в котором рассматривается фотон, априори не очевидно, что она равна 3. Вообще говоря, можно допустить, что фотон находится в четырехмерном пространстве Минковского, тогда формула (74) была бы другой, что не совпадало бы с экспериментом. Можно лишь утверждать, что размерность частоты выбросов отрицательна.

Приведем общее определение отрицательной размерности.

Пусть имеется компакт $M_{t_{0}}$ хаусдорфовой размерности $t_{0}$, являющийся элементом $t$-параметрической шкалы вложенных друг в друга компактов $0<t \leqslant \infty$. Две шкалы будем называть эквивалентными относительно $M_{t_{0}}$, если у них совпадают компакты при $t \geqslant t_{0}$. Будем говорить, что компакт $M_{t_{0}}$ является дыркой в этом множестве эквивалентости шкал, а $-t_{0}$ есть отрицательная размерность этого класса эквивалентности.

Рассмотрим несколько примеров. 
Червяк проедает в стволе дерева сложную извилистую дорожку-дырку. Она настолько меньше ствола, что мы можем считать, что толщина ствола бесконечно велика по сравнению с дыркой. Размерность проволочки той же толщины и точно такой же "извилистости" мы можем определить независимо от окружающей среды, а дырку, проделанную червячком, нельзя рассматривать вне ствола, хотя обе размерности совпадают по абсолютной величине.

В теории кристаллов Френкеля дырка - это отсутствующий электрон, она ведет себя как частица, двигающаяся вдоль кристалла, но в отличие от электрона она не может существовать вне кристалла - в пустоте дырки нет. Точно так же дырка в нашем понимании не может существовать вне достаточно большой шкалы, в которой она "проделана".

Реально фрактальная размерность дырки, трещины в горной породе и т.п. зависит от масштаба, в котором “наш глаз" различает точки. Так, береговая линия будет иметь фрактальный характер, когда мы ее наблюдаем с самолета. Если же мы находимся непосредственно на берегу, то эта линия будет гладкой и ее размерность будет равна размерности гладкой линии, т.е. единице.

Если мы рассматриваем трещины в бетоне [23], то размерность зависит от разрешающей способности прибора, через который мы наблюдаем трещину. Обозначим линейную разрешающую способность прибора через $\kappa$. Обычное определение размерности Хаусдорфа основывается на стремлении к нулю объемов некоторых шаров, на практике, как правило, трехмерных. Это означает, что объем $\Omega$ рассматриваемого металла должен быть много больше, чем $\kappa^{3}$. Таким образом, приведя задачу к единичному объему, мы получим, что объем хаусдорфова шара ${ }^{2)}$ стремится к нулю при $\Omega \rightarrow \infty$.

Далее возникает вопрос, как в конкретных задачах в случае щелей понимать шкалу компактов, размерность которых увеличивается до бесконечно большой величины. Объем металла или горной породы трехмерный. В каком смысле понимать шкалу размерности?

Рассмотрим газ в трехмерном сосуде. Каждая молекула газа движется, число молекул составляет $\approx 10^{23}$. Если рассматривать движение каждой молекулы, то, как правило, можно считать размерность равной $10^{69}$. С другой стороны, можно рассматривать все молекулы как точки трехмерного пространства. И если еще учесть их перемещение за время $t$, то мы можем их рассматривать в четырехмерном пространстве. Именно эта двойственность позволила нам перейти от уравнений Ньютона каждой молекулы к уравнениям полевидной гидродинамики всего газа [25].

Если мы зафиксировали два момента времени расположения молекул газа в $10^{69}$. мерном пространстве, т.е. рассматриваем расположение всех $10^{23}$ молекул как одну точку в первый момент времени и как другую точку во второй момент времени, то мы можем определить естественное расстояние между этими точками, т.е. метрику. Между этой метрикой и метрикой в трехмерном пространстве можно построить шкалу метрик, объединяя различные группы частиц.

2) Т.е. шара, который используется в определении размерности Хаусдорфа (см. [24]). 
Бетон, как и горные породы, с точки зрения механики (теории упругости) и тех масштабов, которые характерны для этой науки, состоят из "зерен” достаточно малого (но не атомного) объема. Эти “зерна" могут быть разной величины, и мы можем их множество считать точкой в пространстве достаточно большой размерности [26]. Именно такая трактовка позволяет нам перейти к общему определению фрактальной щели как дырки и увязать абстрактное математическое определение с реальными механическими объектами.

Распространение дырки-волны особенно наглядно при изучении волн упругости в средах, в которых модуль Юнга на сжатие достаточно велик, а на растяжение равен нулю. В частности, таким свойством обладает песок. Волны-дырки подробно изучены нами в случае одной пространственной координаты (см. приложение в книге [27]).

Рассмотрим теперь простейшие примеры мер в общем случае для $n$-мерных пространств. Пусть $S_{n}-n$-мерный шар радиуса $r$. В сферических координатах его объем $\mu\left(S_{n}\right)$ равен

$$
\text { const } \cdot \int_{0}^{1} r^{n-1} d r=\text { const } \cdot r^{n} \text {, }
$$

где $r^{n-1}-$ плотность.

Оператор умножения на координату двойственен в смысле преобразования Фурье оператору дифференцирования. Поэтому мы можем говорить о двойственных $n$ раз дифференцируемых функциях пространства Соболева $W_{2}^{n}$. "Сопряженным" к такому пространству является пространство $W_{2}^{-n}$ обобщенных по Соболеву функций.

Точно так же мы можем определить пространство $W_{2}^{s}$ для нецелого $s$ как "скалярно" сопряженное к $W_{2}^{-s}$.

Аналогично можно поступить с плотностью или весом $r^{s}$ и $r^{-s}$, используя, например, для представления функций из $W_{2}^{s}$ ядро Рисса.

Приведем пример пространств нецелой положительной размерности с мерой Хаapa $r^{\sigma}$, где $0 \leqslant \sigma \leqslant 1$. На отрезке $0 \leqslant x \leqslant 1$ существует шкала $0 \leqslant \sigma \leqslant 1$ канторовой пыли с мерой Хаара, равной $x^{\sigma}$, отрезка $(0, x)$, подобного всему данному множеству канторовой пыли. Прямое произведение этой шкалы на евклидов куб размерности $k-1$ даст всю шкалу $k+\sigma$, где $k \in \mathbb{Z}, \sigma \in(0,1)$. Именно относительно этой шкалы мы рассматриваем пространство отрицательной размерности $-D=-k-\sigma$.

Кратности $q_{i}$ являются аналогом плотностей в интегральной формуле и, следовательно, связаны с размерностью. В пределе при переходе от сумм к интегралам в формуле (47) мы получим, как это было показано в работе [14], плотности $r^{n-1}$. В этом смысле важно понять, как возникают эти плотности из рядов, т.е. из дискретного аналога. Тем самым мы как бы квантуем эти интегральные плотности: например, квантование меры Хаара канторовой пыли, равной $x^{\sigma}$, имеет вид

$$
q_{i}(D)=\mathrm{const} \cdot \frac{\Gamma(i+\sigma)}{\Gamma(i+1) \Gamma(\sigma+1)},
$$

где значению $x$ отвечает дискретный параметр, принимающий целые значения. 
Благодарности. Автор выражает благодарность Г. В. Ковалю и В.Е. Назайкинскому за плодотворные дискуссии.

\section{Список литературы}

[1] А.Н. Колмогоров, ППИ, 5:3 (1969), 3-7.

[2] В. В. Вьюгин, В.П. Маслов, ППИ, 39:4 (2003), 71-87.

[3] G. Shafer, V. Vovk, Probability and Finance. It's Only a Game!, Wiley, Chichester, 2001.

[4] Д. А. Молодцов, Теория мягких множеств, УРРС, М., 2004.

[5] B. de Finetti, Theory of Probability, Wiley, N.Y., 1990.

[6] В. П. Маслов, В.П. Мясников, В.Г. Данилов, Математическое моделирование аварийного блока Чернобыльской АЭС, Наука, М., 1987.

[7] А.Н. Колмогоров, Юбилейное издание к 100-летию А. Н. Колмогорова. В трех книгах. Книга вторая, Физматлит, М., 2003.

[8] Р. Е. Пайерлс, Законы природъ, Физматгиз, М., 1962.

[9] Р. Кубо, Термодинамика, Мир, М., 1970.

[10] Л. Л. Ландау, Е. М. Лифшиц, Квантовая механика, Гостехиздат, М., 1948.

[11] Н. Н. Боголюбов, Изв. АН СССР. Сер. физ., 11:1 (1947), 77-90.

[12] Н. Н. Боголюбов, Избранные труды. В трех томах, т. 1, 2, Наукова думка, Киев, 1970.

[13] V. M. Long, Maslov-type index, degenerate critical points, and asymptotically linear hamiltonian systems, Science Press, Beijing, 1990; K. Hongo, Yu. Ji, Radio Science, 22 (1987), 357-366.

[14] В.П. Маслов, Матем. заметки, 80:6 (2006), 856-863.

[15] В.П. Маслов, Матем. заметки, 78:6 (2005), 870-877.

[16] В. П. Маслов, Матем. заметки, 78:3 (2005), 377-395.

[17] В. П. Маслов, Матем. заметки, 80:2 (2006), 220-230.

[18] М. В. Федорюк Метод перевала, Наука, М., 1977.

[19] Г. В. Коваль, Частное сообщение, 2006.

[20] M. Gromov, Asymptotic invariants of infinite groups. Geometric Group Theory, vol. 2, Cambridge University Press, 1993.

[21] Л. Д. Ландау, Е. М. Лившиц, Статистическая физика, Наука, М., 1976.

[22] В. П. Маслов, Т. В. Маслова, Матем. заметки, 80:5 (2006), 718-732.

[23] Р. В. Гольдштейн, А. Б. Мосолов, ПММ, 56:4 (1992), 663-671; Докл. РАН, 329:4 (1993), 429-431.

[24] Б. Мандельброт, Фракталъная геометрия природы, Институт компьютерных исследований, М., 2002.

[25] В. П. Маслов, П.П. Мосолов, Изв. АН СССР. Сер. матем., 42:5 (1978), 1063-1100; В.П. Маслов, Квантование термодинамики и ультравторичное квантование, Институт компьютерных исследований, М., 2001.

[26] R. H. Dauskardt, F. Haubensak, R. O. Ritchie, Acta Metallurgica et Materialia, 38 (1990), 143-159; V.E. Saouma, C. C. Barton, N. A. Gamabeldin, Eng. Fract. Mech., 35 (1990), $47-53$.

[27] V.P. Maslov, P. P. Mosolov, Nonlinear wave equations perturbed by viscous terms, Walter de Gruyter, Berlin, N. Y., 2000. 